\title{
The Beneficial Role of Thiamine in Parkinson's Disease: Preliminary Report
}

\author{
Khanh vinh quoc Luonga, b, Lan Thi Hoang Nguyen ${ }^{\mathrm{a}}$
}

\begin{abstract}
Parkinson's disease (PD) is the second most common form of neuro-degeneration in the elderly population. PD is clinically characterized by tremors, rigidity, slowness of movement and postural imbalance. A significant association has been demonstrated between PD and low levels of serum thiamine. Five PD patients presented with stone face, right-hand tremors, Parkinsonian gait and bradykinesia with occasional freezing. Two patients presented with sialorrhea and the plasma transkelosase activity was low in one patient. All of the patients received 100 - $200 \mathrm{mg}$ daily doses of parenteral thiamine. Within days of thiamine treatment, the patients had smiles on their faces, walked normally with longer steps, increased their arm swings, and experienced no tremors or sialorrhea. Three patients did not require carbidopa and levodopa without effects on their movements. Thiamine may benefit to PD. Further investigation of thiamine in PD patients is needed.
\end{abstract}

Keywords: Thiamine; Transketolase; Parkinson's disease; Movement disorders

\section{Introduction}

Parkinson's disease (PD) is a movement disorder characterized by tremor, rigidity, akinesia, and loss of posture reflexes, which leads to immobility and frequent falls. PD results from the selective loss of dopaminergic (DA) neurons in the substantia nigra (SN) of the brain. Recent studies highlight a possible relationship between thiamine and PD. Thiamine may be beneficial for PD patients. Lower central nervous system (CSF)-free thiamine levels were noted in PD patients com-

\footnotetext{
Manuscript accepted for publication August 24, 2012

${ }^{a}$ Vietnamese American Medical Research Foundation, Westminster, California, USA

${ }^{\mathrm{b}}$ Corresponding author: Khanh vinh quoc Luong, FACP, FACE, FACN, FASN, FCCP, and FACAAI (SC), 14971 Brookhurst St., Westminster, CA.92683, USA. Email: Lng2687765@aol.com
}

doi: http://dx.doi.org/10.4021/jnr145e pared with the controls [1]. In parkinsonism-dementia patients, thiamine-pyrophosphatase (TPP) activity was found to be significantly reduced in the frontal cortex [2]. In addition, Gold et al [3] reported that $70 \%$ and $33 \%$ of their PD patients had low plasma and red blood cell (RBC) thiamine levels, respectively. Starvation TD encephalopathy may also induce symmetrical lesions in the SN [4]. These findings suggest that thiamine might have a role in dopaminergic neuron activity. Interestingly, parenteral thiamine administration was used successfully in 9 non-alcoholic patients who presented with acute neurological disorders [5]. Administration of the combination of thiamine and acetazolamide reportedly reduced the Abnormal Involuntary Movement Scale (AIMS) and the Simpson-Angus Neurological Rating Scale (ANRS) scores of patients with tardive dyskinesia and parkinsonism symptoms [6]. In our previous publication, we discussed a number of genetic factors that link thiamine to PD pathology, including the DJ-1 gene, excitatory amino acid transporters (EAATs), the $\alpha$-ketoglutarate dehydrogenase complex (KGDHC), coenzyme Q10 (CoQ10 or ubiquinone), lipoamide dehydrogenase (LAD), chromosome 7, transcription factor p53, the renin-angiotensin system (RAS), heme oxygenase-1 (HO-1), and poly(ADP-ribose) polymerase-1 (PARP-1) gene [7]. In this paper, we report on the role of thiamine in PD patients.

\section{Case Report}

\section{Case 1}

The patient is a 76-year-old male, who has been diagnosed with PD for 8 years. He presented with rigidity, inability to close his mouth, sialorrhea, mask-like facies with infrequent blinking, and his eyelids dropped bilaterally. Additionally, he could not stand or walk alone. He is on carbidopa plus levodopa $(25 / 100$, one tablet twice a day) and benztropine mesylate ( $1 \mathrm{mg}$ tablet twice a day). His blood test was remarkable for transketolase activity less than $2.0 \mathrm{nmol} / \mathrm{L}$ (normal, 8.0 - 30). A brain scan showed cortical atrophy and multiple nonspecific foci in the bilateral subcortical and deep white matter. After being given parenteral thiamine (100 mg daily) for 9 days, he could stand up unassisted and walks with 
normal associated movements in the arms. His eyes opened widely and smiling face. He experienced no sialorrhea. Unfortunately, he had an accident that resulted in a hip fracture, and the follow up ceased.

\section{Case 2}

The patient is a 74-year-old retired male physician, who has been diagnosed with PD for 7 years and presented with stone face with infrequent blinking, tremor of the right hand, loss of the normal associated movements in the arms during walking, walked-assisted Parkinsonian gait and bradykinesia with occasional freezing. He was on carbidopa plus levodopa (25/100, one tablet twice a day) and rasagiline 0.5 $\mathrm{mg}$ daily. He was prescribed with parenteral (200 mg daily). On the second day, he smiled and was able to walk longer steps without a walker and increased his arm swings. After 10 days of treatment, carbidopa plus levodopa and rasagiline were discontinued without any effect on his movement.

\section{Case 3}

The patient is a 68-year-old male who has been diagnosed with PD for 3 years and presented stone face with infrequent blinking, tremor of both hands, Parkinsonian gait and bradykinesia with occasional freezing. His memory had decreased over the last 2 years. He showed mild memory loss by the annotated mini mental state examination (AMMSE). His medications were carbidopa plus levodopa (25/100, one tablet twice a day). Computerized tomography (CT) of the brain revealed cortical atrophy. His plasma thiamine was $9.0 \mathrm{nmol} / \mathrm{L}$ (normal, 4.5 - 15.1). He was treated with parenteral thiamine $200 \mathrm{mg}$ daily. On the fourth day, he smiled and walked normally with longer steps, and increased his arm swings. His AMMSE score returned to normal. After 2 weeks of thiamine treatment, he was taken off carbidopa plus levodopa without any effect on his movement.

\section{Case 4}

The patient is a 65-year-old male, who has been diagnose with PD for 8 years and presented stone face with infrequent blinking, tremor of both hands, difficulty pronouncing words, constant sialorrhea, Parkinsonian gait and bradykinesia with occasional freezing. His plasma thiamine was $6.0 \mathrm{nmol} / \mathrm{L}$ (normal, 4.5 - 15.1). His head CT scan revealed diffuse calcifications throughout the bilateral basal ganglia, caudate nucleus, bilateral occipital gyri, and bilateral cerebellum. He was treated with parenteral thiamine $200 \mathrm{mg}$ daily. On the fourth day, he smiled and walked normally with longer steps, and increased his arm swings; no tremors or sialorrhea were reported. After 10 days of thiamine treatment, he was taken off carbidopa plus levodopa without any effect on his movement.

\section{Case 5}

The patient is an 82-year-old male who has been diagnosed with PD for 16 years and presented with stone face, tremor of the right hand, walked-assisted Parkinsonian gait and bradykinesia with occasional freezing. His medications were carbidopa plus levodopa $(25 / 100$, one tablet three times a day), stalevo 100 (carbidopa $25 \mathrm{mg}$, levodopa $100 \mathrm{mg}$, and entacapone $200 \mathrm{mg}$, one tablet a day), ropinirole extended release (12 $\mathrm{mg}$ a day), and rasagiline (1 $\mathrm{mg}$ daily). His whole-blood thiamine was $109 \mathrm{nmol} / \mathrm{L}$ (normal, 87 - 280). He was treated with parenteral $100 \mathrm{mg}$ daily. On the fourth day, he smiled and walked with longer steps without a walker, increased his arm swing and no longer experienced hand tremor. Unfortunately, this patient was lost to follow-up.

\section{Discussion}

Note that our PD patients improved dramatically in a short time with thiamine supplements. Days after thiamine treatment, they smiled and walked normally with longer steps, and increased arm swings, and no tremors or sialorrhea was reported. Three patients did not receive carbidopa plus levodopa and cessation of those medications did not effect on their movements. The most effective treatment for PD is levodopa in combination with a peripheral decarboxylase inhibitor (carbidopa or benserazide). In a murine model, dopamine has been reported to suppress mouse-killing aggression (muricide) induced by a thiamine-deficient (TD) diet [8]. This suppressive effect can be potentiated with carbidopa [9]. Patients with PD who have undergone levodopa therapy have significantly higher cerebrospinal fluid (CSF) levels of thiamine diphosphate (TDP) and total thiamine than those patients who were not treated with this drug [1]. Moreover, thiamine deficiency can decrease the concentration of dopamine in the striatum, whereas animals fed on a diet that contained 5\% ethanol exhibit increased dopamine turnover [10]. In an experimental TD study, a region-specific vesicular dysfunction (i.e., decreased levels of dopaminergic metabolites) was observed [11]. Dopamine release ii induced by intrastriatal administration of TPP or TDP (up to $1400 \%$ and $249 \%$ of the basal levels, respectively), reduced dopamine levels in the striatum may occur in cases of thiamine deficiency [12].

Sialorrhea is a disabling complication of advanced PD, and it interferes with PD patient's abilities to speak, eat and socially interact with other people. Oral anticholinergic and botulinum toxin have been used as treatment, but the medications provided limited results that usually occurred weeks after treatment [13] and caused side effects. Interestingly, for two patients with sialorrhea, their symptoms disappeared after thiamine treatment.

Thiamine has also been implicated in PD via its effects 
on L-type voltage-sensitive calcium channels (L-VSCC), matrix metalloproteinases (MMPs), prostaglandins (PGs), cyclooxygenase-2 (COX-2), reactive oxygen species (ROS), and nitric oxide synthase (NOS). These factors have been attributed to the pathogenesis of PD [7].

Gastrointestinal dysfunction is common in PD patients, and it potentially affects the therapeutic intervention [14]. Gastric emptying has been reported to be frequently delayed in PD patients [15]. Decreased non-mediated uptake across the enterocyte brush border membrane was demonstrated in PD patients [16]. In addition, the intestinal absorption of thiamine is sufficient in young people but may be reduced with age [17]. A single oral dose of thiamine above $2.5 \mathrm{mg}$ is mostly unabsorbed in humans [18, 19]. Baker et al [20] demonstrated that only the intramuscular administration of thiamine was able to correct thiamine deficiencies in subjects over age 60 . Sasaki et al [21] reported a case study of a patient with a thiamine deficiency and psychotic symptoms. Only repeated intravenous administration of thiamine ameliorated the patient's condition. Furthermore, the patient responded rapidly to large doses of parental thiamine during the early stages of thiamine-deficient encephalopathy (namely Wernicke's encephalopathy). The initial dose of thiamine is usually $100 \mathrm{mg}$ two to three times daily for 1 to 2 weeks. Parental administration of thiamine was also used successfully in patients with general anxiety disorders [22].

\section{Conclusions}

Thiamine may have a beneficial role in PD. Further investigation of thiamine in PD patients is needed.

\section{Conflict of Interest Statement}

The authors, Dr. Khanh vinh quoc Luong and Dr. Lan Thi Hoang Nguyen, report no competing interests.

\section{Ethical Approval}

Not required.

\section{Funding}

The authors, Dr. Khanh vinh quoc Luong and Dr. Lan Thi Hoang Nguyen, received no funding for this study.

\section{References}

1. Jimenez-Jimenez FJ, Molina JA, Hernanz A, Fernandez-Vivancos E, de Bustos F, Barcenilla B, Gomez-
Escalonilla C, et al. Cerebrospinal fluid levels of thiamine in patients with Parkinson's disease. Neurosci Lett. 1999;271(1):33-36.

2. Laforenza U, Patrini C, Poloni M, Mazzarello P, Ceroni M, Gajdusek DC, Garruto RM. Thiamin mono- and pyrophosphatase activities from brain homogenate of Guamanian amyotrophic lateral sclerosis and parkinsonismdementia patients. J Neurol Sci. 1992;109(2):156-161.

3. Gold M, Hauser RA, Chen MF. Plasma thiamine deficiency associated with Alzheimer's disease but not Parkinson's disease. Metab Brain Dis. 1998;13(1):43-53.

4. Kalidass B, Sunnathkal R, Rangashamanna DV, Paraswani R. Atypical Wernicke's encephalopathy showing involvement of substantia nigra. J Neuroimaging. 2012;22(2):204-207.

5. Merkin-Zaborsky H, Ifergane G, Frisher S, Valdman S, Herishanu Y, Wirguin I. Thiamine-responsive acute neurological disorders in nonalcoholic patients. Eur Neurol. 2001;45(1):34-37.

6. Cowen MA, Green M, Bertollo DN, Abbott K. A treatment for tardive dyskinesia and some other extrapyramidal symptoms. J Clin Psychopharmacol. 1997;17(3):190193.

7. Lu'o'ng K, Nguyen LT. Thiamine and Parkinson's disease. J Neurol Sci. 2012;316(1-2):1-8.

8. Tadano T, Abe Y, Morikawa Y, Asao T, Hozumi M, Takahashi N, Tan-no K, et al. Involvement of dopaminergic neurons in mouse-killing aggression in rats. Methods Find Exp Clin Pharmacol. 1997;19(8):527-531.

9. Onodera K. Effects of decarboxylase inhibitors on muricidal suppression by L-dopa in thiamine deficient rats. Arch Int Pharmacodyn Ther. 1987;285(2):263-276.

10. Sjoquist B, Johnson HA, Neri A, Linden S. The influence of thiamine deficiency and ethanol on rat brain catecholamines. Drug Alcohol Depend. 1988;22(3):187193.

11. Mousseau DD, Rao VL, Butterworth RF. Vesicular dysfunction during experimental thiamine deficiency is indicated by alterations in dopamine metabolism. Eur J Pharmacol. 1996;317(2-3):263-267.

12. Yamashita H, Zhang YX, Nakamura S. The effects of thiamin and its phosphate esters on dopamine release in the rat striatum. Neurosci Lett. 1993;158(2):229-231.

13. Chinnapongse R, Gullo K, Nemeth P, Zhang Y, Griggs L. Safety and efficacy of botulinum toxin type B for treatment of sialorrhea in Parkinson's disease: a prospective double-blind trial. Mov Disord. 2012;27(2):219-226.

14. Pfeiffer RF. Gastrointestinal dysfunction in Parkinson's disease. Lancet Neurol. 2003;2(2):107-116.

15. Heetun ZS, Quigley EM. Gastroparesis and Parkinson's disease: a systematic review. Parkinsonism Relat Disord. 2012;18(5):433-440.

16. Davies KN, King D, Billington D, Barrett JA. Intestinal permeability and orocaecal transit time in elderly 
patients with Parkinson's disease. Postgrad Med J. 1996;72(845):164-167.

17. Baum RA, Iber FL. Thiamin--the interaction of aging, alcoholism, and malabsorption in various populations. World Rev Nutr Diet. 1984;44(85-116.

18. Friedemann TE, Kmieciak TC, et al. The absorption, destruction, and excretion of orally administered thiamin by human subjects. Gastroenterology. 1948;11(1):100114.

19. Morrison AB, Campbell JA. Vitamin absorption studies. I. Factors influencing the excretion of oral test doses of thiamine and riboflavin by human subjects. J Nutr.
1960;72(435-440.

20. Baker H, Frank O, Jaslow SP. Oral versus intramuscular vitamin supplementation for hypovitaminosis in the elderly. J Am Geriatr Soc. 1980;28(1):42-45.

21. Sasaki T, Yukizane T, Atsuta H, Ishikawa H, Yoshiike T, Takeuchi T, Oshima K, et al. [A case of thiamine deficiency with psychotic symptoms--blood concentration of thiamine and response to therapy]. Seishin Shinkeigaku Zasshi. 2010;112(2):97-110.

22. Luong K, Nguyen L. The impact of thiamine treatment on generalized anxiety disorder. International Journal of Clinical Medicine, 2011;2:439-443. 